Paper submitted for presentation at the 1988 International Reactor Physics Conference, September 18-21, 1988, in Jackson Hole, Wyoming.

CONF-880911--25

DE89 003887

ADVANCED METHODS COMPARISONS OF REACTION RATES IN THE
PURDUE FAST BREEDER BLANKET FACILITY

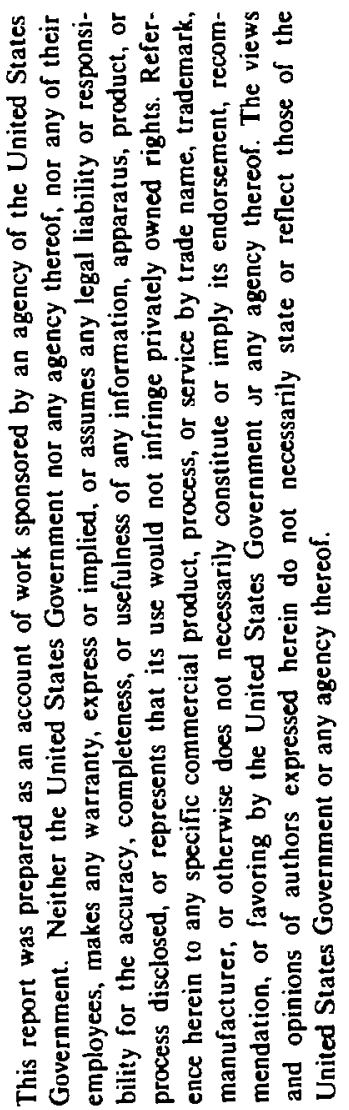

R.N. Hill

\author{
Argonne National Laboratory \\ Applied Physics Division \\ 9700 S. Cass Avenue \\ Argonne, IL 60439
}

and

K.0. Ott

School of Nuclear Engineering

Purdue University

West Lafayette, IN 47907

\begin{abstract}
The submitted manuscript has been outhored
by contractor of the U.S. Government

under contract No. W-31-109ENG-38.

Accordingly, the U.S. Government retains

nonexclusive, royalty-fres license to publish

or reproduce the publithed form of this

contribution, or allow others to do $\infty$, for

U. S. Government purposes.
\end{abstract}

*Work supported by the U.S. Department of Energy, Nuclear Energy Programs under Contract W-31-109-ENG-38. 


\title{
ADVANCED METHODS COMPARISONS OF REACTION RATES IN THE PURDUE FAST BREEDER BLANKET FACILITY
}

\author{
R. N. Hill* and K. O. Ott** \\ *Applied Physics Division, Argonne National Laboratory \\ Argonne, Illinois 60439 U.S.A. \\ **School of Nuclear Engineering, Purdue University \\ West Lafayette, Indiana 47907 U.S.A.
}

\begin{abstract}
A review of worldwide results revealed that reaction rates in the blanket region are generally underpredicted with the discrepancy increasing with penetration; however, these results vary widely. Experiments in the large uniform Purdue Fast Breeder Blanket Facility (FBBF) blanket yield an accurate quantification of this discrepancy. Using standard production code methods (diffusion theory with 50 group cross sections), a consistent Calculated/Experimental (C/E) drop-off was observed for various reaction rates. A $50 \%$ increase in the calculated results at the outer edge of the blanket is necessary for agreement with experiments.

The usefulness of refined group constant generation utilizing specialized weighting spectra and transport theory methods in correcting this discrepancy was analyzed. Refined group constants reduce the discrepancy to half that observed using the standard method. The surprising result was that transport methods had no effect on the blanket deviations; thus, transport theory considerations do not constitute or even contribute to an explanation of the blanket discrepancies. The residual blanket $C / E$ drop-off (about half the standard drop-off) using advanced methods must be caused by some approximations which are applied in all current methods.
\end{abstract}

\section{INTRODUCTION}

The primary focus of fast reactor research has always been the core region because of its importance for criticality, power production, and safety. Initial fast reactor designs consisted of a uniform core region surrounded by a thick radial blanket. The main purpose of the blanket in these designs was to capture neutrons leaking from the core and breed plutonium. Therefore, modeling and analysis of the blanket region have received little attention in the past. Thus, inaccurate physics predictions in the blanket are common and have not been resolved. 
Modern designs have elaborate ex-core regions which serve multiple purposes. The predictions for these complicated advanced designs are not only more difficult than the ones for the earlier simple blankets, they are also more important. Physics predictions in the outer regions are considerably more complicated than core predictions because of the transitory nature of the neutron fiux and spectra.

Blanket measurements have been performed at many fast neutron critical facilities worldwide. Diffusion or transport theory predictions have been compared to these measurements. Several typical examples are presented in Refs. 1-5. These results exhibit a wide variation; however, the majority of the results indicate a $\mathrm{C} / \mathrm{E}$ "drop-off" within the blanket. This drop-off must be quite generic as it has no apparent relation to the details of the composition, the particular reaction, the experimental technique, or the computational method.

A more accurate quantification of blanket discrepancies is obtained from experiments in the large uniform blanket at the Purdue University Fast Breeder Blanket Facility ${ }^{6}$, FBBF (see Section II). The FBBF is a subcritical source driven facility designed specifically to perform benchmark fast reactor blanket measurements. The experimental program includes absolute measurements using activation analysis, fission track recording, and gamma heating methods as described in Refs. 7-10. Blanket predictions were calculated using two-dimensional, fifty group diffusion theory and compared to experimental results. The major result is a fairly accurate quantification of the overall $\mathrm{C} / \mathrm{E}$ ratio drop-off for reaction rates in the blanker which is shown consistently by three different experimental methods.

The $\mathrm{C} / \mathrm{E}$ deviations found in worldwide results are usually attributed to the calculational methods as well as to the nuclear data. This paper will clarify the calculational improvements possible by application of the most advanced calculational methods to one of the large uniform FBBF blankets. The residual blanket $\mathrm{C} / \mathrm{E}$ drop-off (not accounted for by advanced multigroup predictions) is addressed in an analysis of methodological deficiencies in Ref. 11.

The standard FBBF calculational methods and the associated "standard" C/E drop-off are reviewed in Section II. Section III compares current group constant generation methods as applied to FBBF predictions; an augmentation of the $\mathrm{MC}^{2}$ method is developed which applies special transitory weighting spectra. The group constant comparisons include analyses of elastic downscattering treatment, ENDF-IV and V library versions, VTTAMIN-E group structure, and $\mathrm{MC}^{2}$ versus MINX differences. Section IV addresses the contribution of transport theory predictions (using the code TWODANT) to a reduction of the drop-off; one and two-dimensional transport calculations using standard diffusion group constants will be compared to the diffusion predictions.

\section{FBBF RESULTS}

This paper will address predictions only in the stainless steel cladding side of the second FBBF blanket loading; the results for the other two blanket loadings are quite similar. This blanket has a uniform composition with a $51 \mathrm{~cm}$ radial thickness and $120 \mathrm{~cm}$ height. The detailed FBBF geometry is shown in Refs. 7-10.

As the results obtained in this paper will be compared to the "standard" FBBF results, it is necessary to review the details of the "standard" FBBF calculations. The LIB-IV ${ }^{12}$ cross section library was used for multigroup cross section data. LIB-IV is a 50 group Bondarenko method data set generated from ENDF-IV by MINX ${ }^{13}$ for input into the code $1 D X^{14}$. The 50 group structure is summarized in Table 1. The cross section data are self-shielded and mixed in the computer code 1DX utilizing the Bondarenko self-shielding factor methodology.

All newer group constant generation codes that utilize the Bondarenko self-shielding factor method assume a $1 / E$ weighting spectrum within each of the resonance-region groups. 
This simple within-group weighting spectrum is assumed to be adequate for all group cross sections except for the elastic downscattering cross section. Because of the small energy loss in elastic scattering for heavy isotopes, this downscattering occurs mainly from the lower part of the scattering energy group. Thus, it is desirable to use an approximation for this group cross section which accounts better for the spectrum near the lower group boundary. In 1DX, a downscattering correction is applied by using a linear interpolation of the group values of the elastic energy loss source.

This "slowing-down correction" is applied iteratively using revised scattering sources in each step. The fluxes for each iteration are obtained from a complete spatial calculation. This slowing-down iteration was performed five times as suggested in the 1DX manual.

The macroscopic self-shielded group constants obtained from 1DX were used in the code $2 \mathrm{DB}^{15}$ to perform a two-dimensional diffusion calculation. The microscopic self-shielded group constants obtained from 1DX are used in conjunction with the 2DB neutron fluxes to calculate reaction rates. These reaction rates are calculated for the midplane of the blanket at several radial positions corresponding to the location of experimental blanket pins.

The C/E ratio results for various reaction rate measurements are presented in Ref. 8; for most reactions, the $C / E$ value decreases from 1.0 to about 0.65 across the $51 \mathrm{~cm}$ blanket. In Ref. 7, "bulk" reactions are defined as reactions which exhibit approximately a resonance deformed $1 / v$ cross section; these reactions occur over the entire energy range. Thus, the reaction density of these "bulk" reactions is roughly proportional to the total neutron density. The agreement between bulk (U-238 capture and U-235 fission) and low energy (Au-197) reaction rate results indicates that the low energy end of the spectrum exhibits the same drop-off as the total energy spectrum. Uranium-238 fission is a threshold reaction dependent upon the high energy wing of the spectrum. The $C / E$ ratio exhibits a steeper drop-off early in the blanket for this high energy reaction (see e.g. Ref. 8). After the initial sharp drop-off, the $\mathrm{U}-238$ fission $\mathrm{C} / \mathrm{E}$ ratio follows the U-235 fission drop-off since these reactions are the major source of high-energy neutrons within the blanket (Ref. 9).

TABLE 1. LIB-IV 50 group energy structure

$\begin{array}{cccc}\text { Group } & \begin{array}{c}\text { Upper } \\ \text { Energy } \\ (\mathrm{eV})\end{array} & \begin{array}{c}\text { Lower } \\ \text { Energy } \\ (\mathrm{eV})\end{array} & \text { Lethargy } \\ 1 & 1.9971 \times 10^{7} & 1.0000 \times 10^{7} & 0.6917 \\ 2-7 & 1.0000 \times 10^{7} & 4.9787 \times 10^{5} & 0.5 \\ 8-37 & 4.9787 \times 10^{5} & 2.7536 \times 10^{2} & 0.25 \\ 38-49 & 2.7536 \times 10^{2} & 6.8256 \times 10^{-1} & 0.5 \\ 50 & 6.8256 \times 10^{-1} & 1.0000 \times 10^{-5} & 11.13\end{array}$

\section{GROUP CONSTANT COMPARISONS}

\section{III.A MC ${ }^{2}$ Group Constant Generation}

In the $\mathrm{MC}^{2}-2$ code $^{16}$ one calculates a detailed weighting spectra for a specific composition represented by a zero-dimensional model. For the purpose of these comparisons zero-dimensional $\mathbf{M C}^{2}$ spectra were adapted to represent transition spectra within the two major regions, the transformer and the blanket. The resulting two sets of microscopic group 
constants were also used in the respective neighboring regions.

It is important that a detailed $\mathrm{MC}^{2}$ spectrum accurately models the actual spectrum. If this is not the case, the increased detail may not yield better accuracy. It is a well known fact that blanket spectra become softer with increasing penetration. A fundamental mode calculation yields the asymptotic spectrum; this spectrum is so soft that it would not be physically realized until about $100 \mathrm{~cm}$ of blanket penetration. Conversely, the $\lambda$-mode spectrum is harder than physically realized blanket spectra. Thus, a special method was devised to yield an accurate blanket weighting spectrum:

The fundamental mode solution is achieved by modifying the buckling of the system, this effectively changes the leakage term. Conversely, the $\lambda$-mode solution is achieved by multiplying the fission source with an eigenvalue. This latter solution would greatly increase the fission source in the subcritical blanket region without the corresponding increase in absorption. This artificial modification does not yield a physically realizable spectrum. Thus, a suitable fundamental mode solution is preferred.

A more accurate blanket weighting spectrum was obtained by increasing the U-235 concentration in a fundamental mode calculation. As the U-235 concentration is gradually increased, the fundamental spectum becomes harder simulating the actual spectral transition with increasing proximity to the core. This procedure was performed until the average lethargy of the $\mathrm{MC}^{2}$ fundamental spectrum agreed with the previously calculated standard 2DB spectrum about $20 \mathrm{~cm}$ into the blanket, the mean location for blanket reaction rates. The increased U-235 number density (about 2\% enrichment) will have minimal effects on selfshielding for all other isotopes because of its low enrichment. This spectrum then exhibits the correct flux depression for the broad scattering resonances within a transitory spectrum of the correct overall shape. The U-235 group cross sections were generated separately using the SDX code ${ }^{17}$ methodology which is similar to the Bondarenko method.

The $\mathrm{MC}^{2} 2040$ group library was collapsed, using the detailed weighting spectra, to a 147 group set which is compatible with the LIB-IV energy structure. This 147 group structure was designed to have three equal lethargy width divisions for each of the LIB-IV energy groups; however, because of differing lower and upper boundaries in the $\mathrm{MC}^{2}$ and MINX input libraries, less than three sub-divisions were used for the highest and lowest energy group yielding a 147 group structure. Various codes were written to re-format the $\mathrm{MC}^{2}$ ISOTXS file into an appropriate format for 1DX input; spatial collapsing was then performed with 1DX to obtain a new, independent 50 group cross section set based on ENDF-V and the detailed $\mathrm{MC}^{2}$ weighting spectra.

\section{B Elastic Downscattering Comparison}

The effects of the IDX downscattering modification and subsequent slowing down iteration on this modification were investigated in Ref. 11. One expects a modification of elastic downscattering for this within-group bias to give better results; however, the following results were observed:

- Differences between $N=1$ (single modification) and $N=5$ (iterated) results are negligible indicating that the slowing down iteration is unfruitful for this problem.

- The $\mathbf{N}=0$ (unmodified) results are higher than the $N=1$ predictions with the difference increasing with increasing penetration; thus, the slowing-down correction enlarges the underprediction of calculated results as compared to experiments.

In contrast to the bulk neutron population, the comparison of U-238 fission (the high energy end of the spectrum) indicated a very different behavior. The unmodified group constants exhibit a large overprediction (C/E = 1.15) of the high energy wing leaving the transformer region. Correction of the elastic downscattering group constants is most 
important for the high energy groups because of the large group widths for groups $1-7$ in the LIB-IV structure (see Table 1); thus, the high energy deviations of the $N=0$ results are attributed to the lack of an appropriate downscattering treatment for these wide energy groups.

A more detailed elastic downscattering iteration was proposed by Kidman in $1976 .{ }^{18}$ This improved modification yielded elastic downscattering corrections that were much smaller than the 1DX method. In Kidman's analysis, the 1DX method yielded large changes in the elastic downscattering term (from a factor of 5 increase to a factor of 20 decrease) particularly in resonance groups. Although most of the 1DX modifications appear to be in the right direction, they tend to be too large. Similar excessive modifications were observed for the FBBF downscattering group constants using the 1DX slowing-down iteration.

An evaluation of flux interpolation methods for downscattering in $1977^{19}$ (Kidman, et al.) concluded that no interpolation $(N=0)$ is superior in the 1DX method because of the large changes introduced by the slowing-down correction. The FBBF blanket results presented in Ref. 11 support this conclusion; the unmodified group constant results lead fortuitously to a smaller C/E drop-off across the blanket. However, the deviations in the U-238 fission results demonstrate that better downscattering group constants are needed at the high energy end. A finer high energy group structure would avoid dubious downscattering corrections; and if a correction is applied, it should employ a better method than the one used in 1DX.

\section{III.C ENDF Version $N$ and V Comparison}

Both ENDF-IV and $\mathrm{V}$ nuclear data were available for use in the $\mathrm{MC}^{2}$ code; group constants from each version were generated in an identical manner as described previously. The group constants were then spatially collapsed in 1DX using the FBBF mid-plane geometry. Thus, the only differences in the generation of these group constants is the original ENDF data file.

The top section of Fig. 1 shows the reaction rate differences as a ratio of Version-V predictions to Version-IV predictions. The Version-V data yield slightly higher predictions for U-238 capture and U-235 fission (about 2-3\% higher). However, the C/E drop-off for U238 fission is reduced by about $8 \%$ using the Version-V data.

Major changes in the iron data are probably the cause of the slightly higher bulk reaction rates using ENDF-V. The U-238 fission cross sections are slightly higher for Version-V for the majority of the energy range leading to the higher U-238 fission rates. In addition, the Version- $V \chi$ vector is slightly harder than the Version-IV $\chi$ vector which leads to more neutrons in the threshold groups.

\section{D VITAMIN-E Group Structure Effect}

The VITAMIN-E 174 group library ${ }^{20}$ generated from ENDF-V using MINX is another self-shielding factor group constant set which was developed at Oak Ridge National Laboratory for fusion and shielding applications as well as reactor analysis. VITAMIN-E group constants were processed using the MARS ${ }^{21}$ package; specifically, the BONAMI module was used to generate self-shielded cross sections for the transformer and blanket compositions.

One way to improve the downscattering representation is to utilize a finer group structure. For sufficiently small groups the elastic downscattering will occur almost uniformly across the group. VITAMIN-E has a detailed high energy group structure for application to fusion and other fast neutron flux calculations. In addition, the VITAMIN-E group structure was further refined by modeling the group boundaries around the resonances of several important reactor materials (such as iron) providing a more accurate representation of the resonance structure. The VTTAMIN-E downscattering group constants agreed very well with a 
corresponding set derived from detailed $\mathrm{MC}^{2}$ spectra as shown in Ref. 11.

The VITAMN-E blanket predictions are compared to the standard calculations in the center section of Fig. 1. A consistent increase compared to the standard results is observed in predictions for all three reaction rates with the U-238 fission increase being the largest. These refined predictions explain a considerable part of the C/E drop-offs. The higher reaction rate predictions of VITAMIN-E are the result of a combination of several factors:

- The use of superior downscattering group cross sections yields higher predictions than the standard method. Taking the difference of the $\mathrm{N}=0$ versus standard results (see Sec. III.B) as an indication of the magnitude, improved elastic downscattering matrices yield an increase of about $8 \%$ in the outer blanket bulk predictions.

- The use of ENDF-V group constants yields 2-3\% higher bulk and $8 \%$ higher U-238 fission reaction rate predictions than ENDF-IV in the outer parts of the blanket (see upper part of Fig. 1).

- The straight forward application of the VITAMIN-E group constants yields even higher predictions than the above two effects. These higher predictions are most likely caused by a refined group structure.

\section{III.E Group Constant Generation Differences : $\mathrm{MC}^{2}$ Vs. MINX}

The major difference between the two generation methods, $\mathrm{MC}^{2}$ and $\mathrm{MINX}$, is in the weighting spectra. A $1 / E$ weighting spectrum is used in MINX as compared to a detailed composition dependent spectrum in $\mathrm{MC}^{2}$. The broad resonances are treated explicitly in the $\mathrm{MC}^{2}$ generation scheme; but the NR approximation is applied for all resonances in MINX. A further difference is the spectral weighting of the actinide transpor group constants as discussed below.

To isolate the effects of weighting spectra differences, the $\mathrm{MC}^{2}$ group constants were collapsed to 50 groups using the detailed weighting spectra for the transformer and blanket regions obtained by the method described in Sec. III.A. The 1DX code was run for a two region problem, a transformer and a blanket region, to obtain MINX (LIB-IV) group cross sections self-shielded for the same two regional compositions omitting the slowing-down correction. Thus, these two group constant sets differ only in the use of the group constant processing code, $M I N X$ versus $\mathrm{MC}^{2}$.

Results for the three reaction rates are presented in the lower section of Fig. 1 as ratios of the $\mathrm{MC}^{2}$ to $\mathrm{MINX}$ predictions. The $\mathrm{MC}^{2}$ predictions are lower with the difference compounding through the blanket. The $7-10 \%$ lower predictions for the bulk reactions in the outer blanket indicate that there must be significant differences between these two group constant sets. The large U-238 fission differences are caused by the overprediction of U-238 fission for unmodified LIB-IV group constants. The sudden increase of the U-238 capture and U-235 fission ratios at the end of the blanket is likely not a methodological effect but is probably caused by a group constant inconsistency in the reflector region leading to special effects at the blanket/reflector interface

The MNX and $\mathrm{MC}^{2}$ group constant values were compared in Ref. 11. Two conclusions were drawn:

- Deviations in the fission and absorption group constants are small for the important energy groups and vary such that most of the differences cancel.

- Major differences are indicated in the transport group constant; below group 21 the $\mathrm{MC}^{2}$ transport group constant is consistently 2-10\% higher than the MINX values, increasing up to $40 \%$ for lower energies.

The larger $\mathrm{MC}^{2}$ transport group constants will yield smaller diffusion constants which will reduce the spatia transmission of neutrons in the $\mathrm{MC}^{2}$ predictions. Thus, the difference in 
transport group constants is the probable cause of the lower predictions using the $\mathrm{MC}^{2}$ group constants.

The larger $\mathrm{MC}^{2}$ transpor group constants are consistent with previous results ${ }^{22,23}$; however, previous comparisons ${ }^{23}$ focused on infinite medium and eigenvalue predictions for which transport constants are not used or are relatively unimportant. However, for the blanket transmission problem the transport constants will be crucial data for accurate predictions as shown in Ref. 24.

The cause of these differing transport group cross sections is the use of different weighting spectra within the actinide resonances. The MINX method uses an approximate neutron current, the reciprocal of the total cross section squared, for the transport group constant weighting spectrum of all resonances. In $\mathrm{MC}^{2}$, a neutron flux weighting, the narrow-resonance approximation flux (i.e. reciprocal of the total cross section), is applied for the actinide resonances; a current weighting is applied for the broad scattering resonance transport group constant. This approximation was justified by the fact that for many reactor compositions the contribution of the actinides to the transport group constant is small. However, for the softer spectrum and greater U-238 concentration in a blanket transmission problem large differences in the lower energy groups are quite important and are likely responsible for the indicated differences.

The VITAMIN-E and $\mathrm{MC}^{2} \mathrm{C} / \mathrm{E}$ ratios for U-238 capture are compared to the standard $\mathrm{C} / \mathrm{E}$ curve in Fig. 2. The $\mathrm{MC}^{2}$ predictions are lower than the MINX generated VITAMIN-E predictions likely because of the inaccurate within group weighting of the transpor group constants for actinides.

\section{TRANSPORT AND DIFFUSION COMPARISONS}

For this analysis, transport calculations were performed using the one-dimensional code, ONEDANT $^{25}$, and the two-dimensional code, TWODANT ${ }^{26}$, both developed at Los Alamos National Laboratory. All transport calculations were performed using $S_{\mathbf{8}}$ angular quadrature and $P_{1}$ Legendre expansion scattering matrices. It was shown in Ref. 27 that higher order quadrature and scattering yielded nearly the same results for the FBBF problem.

The one-dimensional FBBF transport problem models the mid-plane FBBF geometry. The transport group cross section is generally not used in transport theory calculations. Therefore, axial leakage corrections $\left(\mathrm{DB}_{z}^{2}\right)$ are inconsistent between transport and diffusion theory; generally the total group cross section is used in place of the transport group cross section in the transport code. Thus, to allow a rigorous comparison of the diffusion and transport results, the axial leakage was disregarded for the one-dimensional comparisons. The neglect of axial leakage effects was subsequently justified by two-dimensional comparisons which yielded virtually the same ratios of diffusion versus transport results in the blanket region. Therefore, only the one-dimensional results are presented.

The one-dimensional transport calculation was run at the fine group level for both cross section sets; VITAMIN-E is a 174 group library and $\mathrm{MC}^{2}$ was used to generate a 147 group library. A one-dimensional transport calculation using a 50 group spatially collapsed $\mathrm{MC}^{2}$ group constant set was also performed. Diffusion calculations were performed for the VITAMIN-E and $\mathrm{MC}^{2}$ group constant sets using the standard 50 group structure in the $2 \mathrm{DB}$ code.

The ratios of the transport to diffusion theory total flux are presented in Fig. 3. The amazing and unexpected result is that the transport and diffusion predictions show good agreement throughout the entire blanket region. In fact, the transport predictions are slightly lower throughout the blanket implying a slightly larger $C / E$ drop-off for bulk reactions. This agreement is explained by the almost linear dependence $\left(P_{1}\right)$ of the angular flux distribution 
on $\mu=\cos \theta$ for this problem, as observed in Ref. 27 .

The transport/diffusion ratios agree very closely for the three group constant sets. Two conclusions can be drawn from this agreement:

- Transport calculations using the $\mathrm{MC}^{2}$ group constants, either 147 or 50 groups, yielded nearly the same total fluxes. This implies that group collapsing leads to very small changes in transport predictions at this fine group level.

- The VITAMN-E and $\mathrm{MC}^{2}$ transport to diffusion total flux ratios are also about the same; thus, the finer high energy structure used in the 174 group VITAMIN-E set has not caused any significant changes in the total flux as compared to the 147 group $\mathrm{MC}^{2}$ structure. However, spectral differences could still be present.

Reaction rate comparisons showed trends for the bulk reactions (U-238 capture and U-235 fission) similar to the total flux results of Fig. 3; transport theory predictions are 3-8\% lower throughout the blanket and do not provide an improvement as compared to diffusion theory.

The high energy end exhibits a different behavior. The transport U-238 fission rate predictions at the transformer/blanket interface are significantly lower than the diffusion predictions which agreed well with experiment; this result is surprising since one would expect transport theory to yield more accurate predictions particularly for high energy neutrons. Since this underprediction was not observed in critical facility $\mathrm{C} / \mathrm{E}$ comparisons, it is suspected that the source geometry of the FBBF leads to some discrepancies in transport predictions near the source. For this paper, it is sufficient to note that the transport predictions do not significantly reduce the general blanket $C / E$ drop-off.

The results do show that transport theory describes the severe transition of the high energy wing in the inner blanket more accurately than diffusion theory. This improved treatment of the initial transition does not lead to significantly higher blanket predictions because of the underprediction of the high energy flux leaving the transformer.

\section{SUMMARY AND CONCLUSIONS}

Worldwide results indicate an underprediction of reaction rates in fast reactor blankets. Experiments in the large uniform FBBF blanket yield an accurate quantification of this $\mathrm{C} / \mathrm{E}$ drop-off. Thus, comparisons of calculations with the most advanced methods have been performed to assess the "residual" of the $C / E$ drop-off which can then be attributed to more generic methodological deficiencies.

Summarizing the comparisons of FBBF results with current methods combining the best group constant generation with the best fiux calculational methods:

- Refined group constant generation can account for nearly one half of the drop-off which resulted from a "standard" 50 group diffusion calculation using LIB-IV group constants.

- Contrary to general expectations, transport theory does not constitute (or even contribute to) an explanation of the blanket C/E drop-off. Transport theory applications actually yield lower FBBF predictions, slightly increasing the drop-off. (Some special effects at the high energy end of the spectrum require further study.)

- The smallest C/E drop-off in this analysis of "state-of-the-art" methods was obtained with the VITAMN-E diffusion results as presented in Fig. 2. These VITAMIN-E results show a residual drop-off from 1.0 to 0.8 for U-238 capture across a $51 \mathrm{~cm}$ uniform blanket (as compared to 1.0 to 0.65 for the "standard" calculations).

In conclusion, the residual blanket $\mathrm{C} / \mathrm{E}$ drop-off must be caused by some other approximations which are applied in all current methods. 


\section{ACKNOWLEDGMENTS}

This work was supported by a contract with the U.S. Department of Energy and under an appointment to the Nuclear Engineering and Health Physics Fellowship program administered by Oak Ridge Associated Universities for the U.S. Department of Energy. The major funding for the construction of the FBBF was provided by the Electric Power Research Institute.

\section{REFERENCES}

1. J. E. Sanders, "The Zero-Power Basis of Fast Reactor Dosimetry," UKAEA, AEEW-R1183 (1978).

2. M. Edelmann, E. A. Fischer, F. Helm, and R. Schroder, "Physics Measurements in the SNEAK Facility on Steam-cooled Fast Reactor Systems with Uranium and Plutonium Fuel," Proc. International Conf. on the Physics of Fast Reactor Operation and Design, British Nuclear Energy Society, 113 (1969).

3. G. Humbert, F. Kappler, M. Martini, G. Norvez, G. Rimpault, B. Ruelle, A. Stanculescu, and W. Scholtyssek, "Parametric Studies on Heterogeneous Cores for Fast Breeder Reactors: The Pre-Racine and Racine Experimental Programs," Nucl. Sci. and Eng. , 87 , (1984).

4. L. A. Alekhin, A. V. Zvonarev A. P. Ivanov, Yu A. Kazanskii, A. V. Karpov, B. V. Koloskov, V. V. Mateev, V. Iv Maatveev, G. B. Pomerantsev, Pshakin, N. V. Skorikov, N. D. Tverdovskii, M. S. Troysnov, E. M. Khalov, and V. S. Shkol'nik, "Mathematical and Experimental Investigations into the Physical Characteristics of the BNL-350 reactor," UKAEA, RISLEY-Trans-3315 (1978).

5. S. Nomoto, H. Yamamoto, and Y. Sekiguchi, "Physics Measurements at the Start-up of JOYO," Proc. IAEA and NEA Symposium on Fast Reactor Physics, Aix-En-Provence, IAEA-SM-244/8, 1, 463 (1979).

6. K. O. Ott, F. M. Clikeman, P. J. Fulford, R. H. Johnson, and O. H. Gailar, "The Purdue Fast Breeder Blanket Facility," Proc. ANS Topical Meeting on Advances in Reactor Physics, Gatlinburg, TN, 1978, CONF-780401, 505, (1978).

7. K. O. Ott, F. M. Clikeman, and G. A. Harms, "Neutron and Gamma-Ray Physics in Fast Reactor Blankets," Nucl. Sci. and Eng. , 88, 1 (1984).

8. F. M. Clikeman, S. C. Mo, K. O. Ott, G. A. Harms, H. P. Chou, and R. H. Johnson, "Neutron Reaction Rate Measurements as Tests of Diffusion Calculations in a Fast Breeder Blanket Facility," Nucl. Tech. , 71 , 341 (1985).

9. H. C. Vital, F. M. Clikeman, and K. O. Ott, "Fission Rates and High-Energy Neutron Fluxes in the Purdue Fast Breeder Blanket Facility," Nucl. Sci. and Eng. , 96 , 102 (1987).

10. T. K. Wang, F. M. Clikeman, and K. O. Ott, "Experimental and Computational Studies of the Gamma-Ray Energy Deposition Rate in the Purdue Fast Breeder Blanket Facility," Nucl. Sci. and Eng. , 98, 262 (1986).

11. R. N. Hill, "Analysis of Deficiencies in Fast Reactor Blanket Physics Predictions," Ph. D. Thesis, Purdue University (1987).

12. R. B. Kidman and R. E. MacFarlane, "LIB-IV, A Library of Group Constants for Nuclear Reactor Calculations," Los Alamos Scientific Laboratory, LA-6260-MS (1976).

13. C. R. Weisbin, P. D. Soran, R. E. MacFarlane, D. R. Harris, R. J. LaBauve, J. S. Hendricks, J. E. White, and R. B. Kidman, "MINX: A Multigroup Interpretation of 
Nuclear X-Sections from ENDF/B," Los Alamos Scientific Laboratory, LA-6486-MS (ENDF-237), (1976).

14. R. W. Hardie and W. W. Little, Jr., "1DX, A One-Dimensional Diffusion Code for Generating Effective Nuclear Cross Sections," Batelle Northwest Laboratory, BNWL954 (1969).

15. W. W. Little, Jr. and R. W. Hardie, "2DB User's Manual-Revision 1," Batelle Northwest Laboratory, BNWL-821-REV1 (1969).

16. H. Henryson II, B. J. Toppel, and C. G. Stenberg, "MC ${ }^{2}-2$ : A Code to Calculate Fast Neutron Spectra and Multigroup Cross Sections," Argonne National Laboratory, ANL8144 (ENDF-239), (1976).

17. W. M. Stacey, Jr., B. J. Toppel, H. Henryson II, B. A. Zolotar, R. N. Hwang, and C. G. Stenberg, "A New Space-Dependent Fast-Neutron Multigroup Cross-Section Preparation Capability," Trans. Am. Nucl. Soc. , 15, 292 (1972).

18. R. B. Kidman, "Spectral Corrections to Elastic-Removal Cross Sections," Trans. Am. Nucl. Soc. , 24,468 (1976).

19. R. B. Kidman, R. E. MacFarlane, and M. Becker, "Flux Interpolation for Elastic Downscatter," Trans. Am. Nucl. Soc. , 26, 580 (1977).

20. R. W. Roussin, "VITAMIN-E: A Coupled 174-Neuton, 38-Gamma-Ray Multigroup Cross-Section Library for Deriving Application-Dependent Working Libraries for Radiation Transport Calculations," Oak Ridge National Laboratory, DLC-113 (1984).

21. "MARS: Collection of Computer Codes for Manipulating Multigroup Cross Section Libraries in AMPX or CCCC Formats," Oak Ridge National Laboratory, PSR-117.

22. R. B. Kidman, R. E. Schenter, R. W. Hardie, and W. W. Little, "The Shielding Factor Method of Generating Multigroup Cross Sections for Fast Reactor Analysis," Nucl. Sci. and Eng. , 48, 189-201 (1972).

23. R. B. Kidman, R. E. MacFarlane, and M. Becker, "Differences Between LASL-and ANL-Processed Cross Sections," Los Alamos Scientific Laboratory, LA-7191-MS (1978).

24. K. O. Ott, K. R. Boldt, and F. M. Clikeman, "Review of Nuclear Physics Uncertainties in Fast Reactor Blankets," Purdue University, PNE-75-103 (1975).

25. R. D. O'Dell, F. W. Brinkley, Jr., and D. R. Marr, "User's Manual for ONEDANT: A Code Package for One-Dimensional, Diffusion-Accelerated, Neutral-Particle Transport," Los Alamos National Laboratory, LA-9184-M (1982).

26. R. E. Alcouffe, F. W. Brinkley, D. R. Marr, and R. D. O'Dell, "User's Guide for TWODANT: A Code Package for Two-Dimensional, Diffusion-Accelerated, NeutralParticle Transport," Los Alamos National Laboratory, LA-10049-M (1984).

27. T. Wang, "Experimental and Computational Studies of the Gamma-Ray Energy Deposition Rate in the Purdue Fast Breeder Blanket Facility," Ph. D. Thesis, Purdue University (1984). 


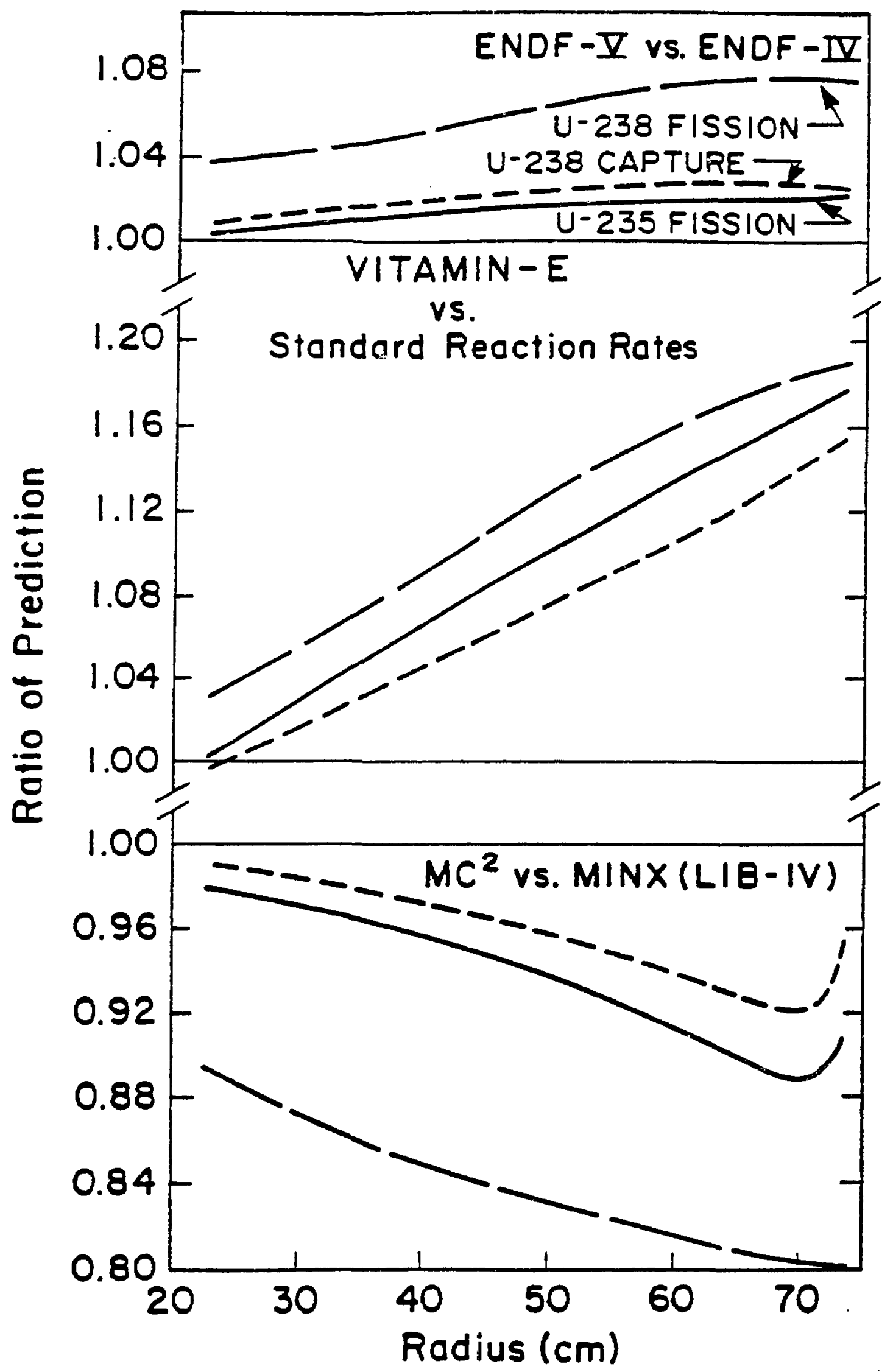

Fig. 1. Reaction rate ratios of various group constant comparisons. 


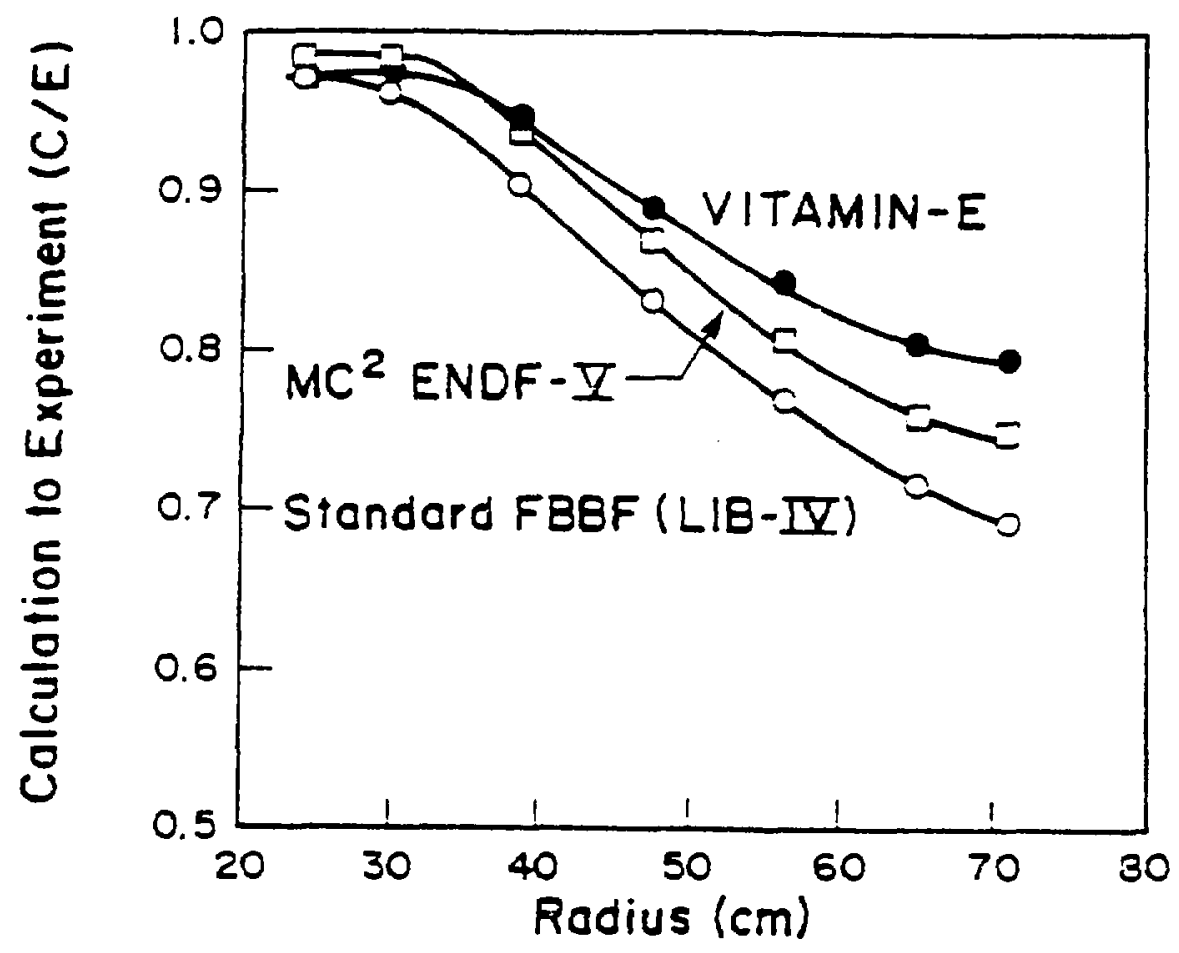

Fig. 2. Comparison of Calculation-to-Experiment (C/E) results for U-238 in the FBBF (Blanket 2-SS) using three different sets of group constants.

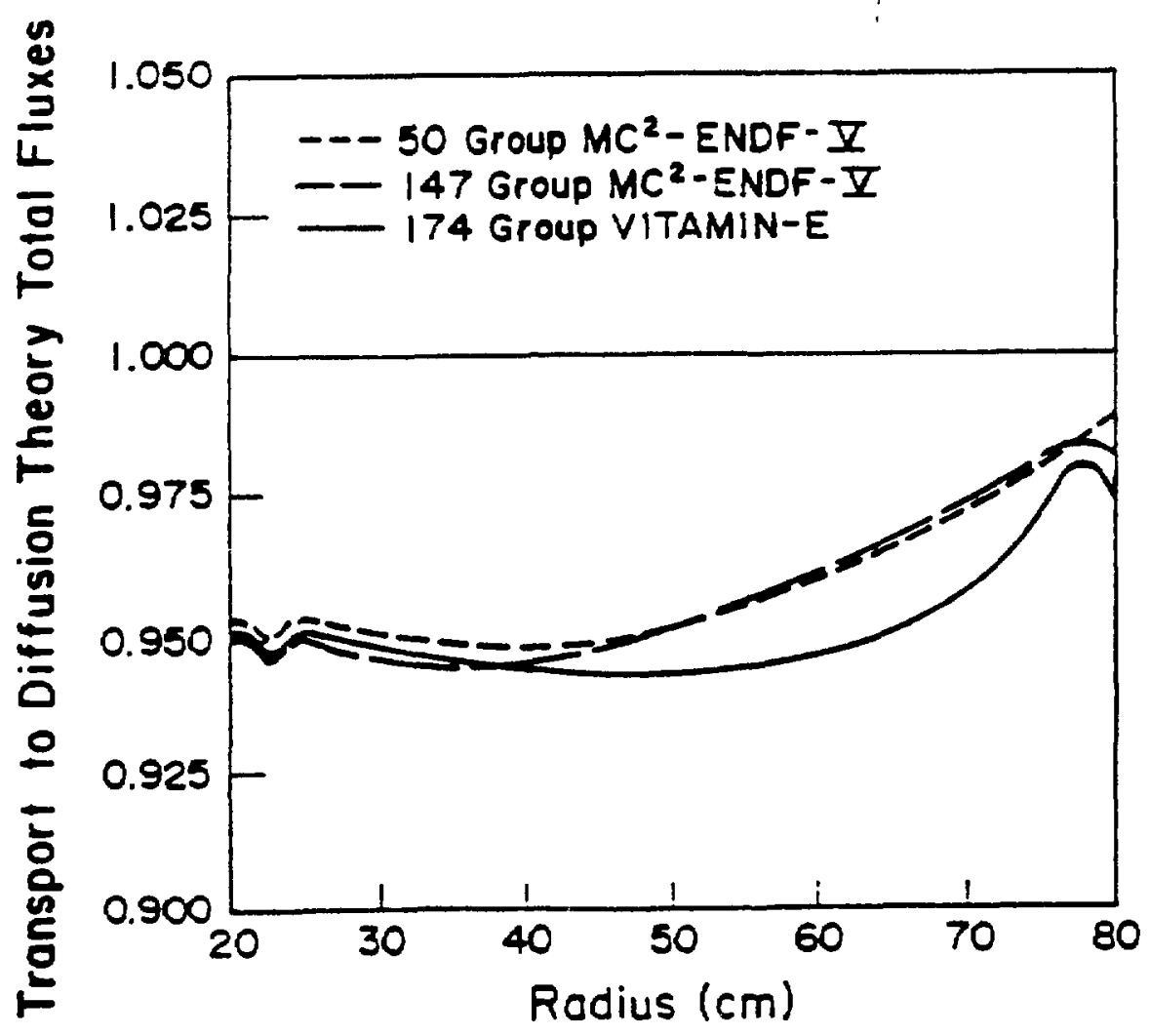

Fig. 3. Comparison of transport $\left(S_{8} P_{1}\right)$ and diffusion theory total fluxes for the FBBF Blanket 2-SS using $M C^{2}$ and VITAMIN-E group constant sets. 